\title{
Numerical and experimental study of a droplet-based PCR chip
}

\author{
S. Mohr ${ }^{1}$, Y-H. Zhang ${ }^{2 *}$, A. Macaskill' ${ }^{1}$, P. J. R. Day ${ }^{3}$, R. W. Barber ${ }^{2}$, N. J. Goddard ${ }^{1}$, \\ D. R. Emerson ${ }^{2}$, P. R. Fielden ${ }^{1}$
}

1. School of Chemical Engineering and Analytical Science, University of Manchester, M60 1QD, UK.

2. Centre for Microfluidics and Microsystems Modelling, CCLRC Daresbury Laboratory, Warrington, Cheshire, WA4 4AD, UK.

3. Centre for Integrated Genomic Medical Research, University of Manchester, Stopford Building, Oxford Road, Manchester, M13 9PT, UK.

\begin{abstract}
A two-temperature continuous-flow PCR polymer chip has been constructed that takes advantage of droplet technology to avoid sample contamination and adsorption at the surface. Samples contained in aqueous droplets are continuously moved by an oil carrier-fluid through various temperature zones, introducing the possibility of real-time quantitative PCR. In the present paper, we investigate many of the factors affecting droplet-based PCR chip design, including thermal mass, flow rate, and thermal resistance. The study focuses particularly on the fluid and substrate temperature distribution within the PCR chip and the droplet residence times in critical temperature zones. The simulations demonstrate that the flow rate strongly affects the temperature field within the carrier-fluid. Above a critical flow rate, the carrierfluid fails to achieve the required temperatures for DNA amplification. In addition, the thermal resistances of the different layers in the chip are shown to have a major impact on the temperature profile in the channel.
\end{abstract}

\section{Keywords}

DNA amplification, PCR chip, continuous flow, droplet, thermal cycling.

\footnotetext{
*Author to whom correspondence should be addressed. Email: y.zhang@dl.ac.uk
} 


\section{Nomenclature}

$\begin{array}{ll}\text { SymbolDescription } & \text { Unit }\end{array}$

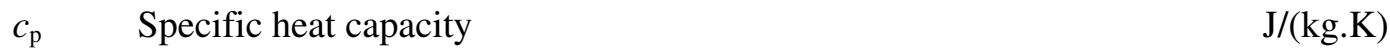

d Droplet diameter $\mathrm{m}$

$d_{1} \quad$ Thickness of cellulose acetate layer $\quad \mathrm{m}$

$d_{2} \quad$ Thickness of polycarbonate layer m

$f_{b} \quad$ Buoyancy force $\quad \mathrm{N}$

$f_{\text {Saff }} \quad$ Saffman lift force $\quad \mathrm{N}$

g Acceleration due to gravity $\mathrm{m} / \mathrm{s}^{2}$

$\begin{array}{lll}k & \text { Thermal conductivity } & \mathrm{W} /(\mathrm{m} . \mathrm{K})\end{array}$

$p$ Pressure N/m

$Q \quad$ Flow rate $\quad \mu 1 / \mathrm{min}$

$R_{1} \quad$ Thermal resistance of acetate layer $\quad \mathrm{m}^{2} . \mathrm{K} / \mathrm{W}$

$R_{2}$ Thermal resistance of polycarbonate layer $\quad \mathrm{m}^{2} . \mathrm{K} / \mathrm{W}$

$R_{3} \quad$ Thermal resistance of natural convection $\quad \mathrm{m}^{2} . \mathrm{K} / \mathrm{W}$

Re Reynolds number -

$T$ Temperature $\quad \mathrm{K}$

$t$ Time $\mathrm{s}$

$\boldsymbol{V}$ Velocity vector $\mathrm{m} / \mathrm{s}$

$V_{\mathrm{r}} \quad$ Relative velocity $\mathrm{m} / \mathrm{s}$

Greek symbols

$\gamma \quad$ DNA amplification efficiency

$\mu \quad$ Dynamic viscosity $\quad$ N.s $/ \mathrm{m}^{2}$

$v \quad$ Kinematic viscosity $\mathrm{m}^{2} / \mathrm{s}$

$\rho$ Density $\mathrm{kg} / \mathrm{m}^{3}$

$\tau \quad$ Shear stress $\quad N / m^{2}$

\section{Subscripts}

1,2,3 Layers 1, 2 and 3 


\section{Introduction}

Since its introduction in 1985, the polymerase chain reaction (PCR) process for amplifying DNA has revolutionized many life science applications and related areas, including clinical diagnoses and medical, biological, and forensic analyses (e.g. Saiki et al. 1985; Auroux et al. 2002, 2004; Vilkner et al. 2004). However, standard PCR has several disadvantages including high consumption of expensive reagents, potential for sample contamination, and a large thermal mass. Recent developments in micro-technology can overcome these problems. For example, miniaturized PCR can achieve rapid heat transfer and quick sample mixing due to the large surface-to-volume ratio. At the same time, sample handling, detection, mixing, and separation can be integrated into a single chip so that the device is easy to operate and interactions between the surface and the sample are minimized, reducing the possibility of sample contamination. Moreover, the thermal cycling time will be significantly reduced and the reagents will be exposed to more uniform temperatures during the PCR process, thereby enhancing the yield. A further attractive feature of miniaturized PCR is its portability, making it useful for in-the-field detection and analysis.

Currently, PCR chips can be classified into two distinct types: well-based PCR chips (e.g. Northrup et al. 1993; Wilding et al. 1994; Belgrader et al. 1999; Gulliksen et al. 2004) and continuous-flow PCR chips (e.g. Nakano et al. 1994; Kopp et al. 1998; Chiou et al. 2001; Chen et al. 2004). In well-based PCR, the sample is injected into the well and the chip is heated and cooled through specific thermal-cycling temperatures. However, the large total thermal mass of the system creates unwanted inertial effects. In contrast, continuous-flow PCR moves the sample through fixed temperature zones to achieve the required thermalcycling. This approach has a smaller thermal inertia because only the sample and associated reagents need to be heated and cooled, rather than the entire chip. This allows rapid thermalcycling and also consumes less energy, making the system more amenable to portable applications and integration into Micro-Total-Analysis-Systems ( $\mu$ TAS).

Continuous-flow PCR chips can be further subdivided into oscillatory devices (Bu et al. 2003; Auroux et al. 2005), closed-loop devices (Liu et al. 2002; West et al. 2002; Sadler et al. 2003; Chen et al. 2004), and fixed-loop devices (Nakano et al. 1994; Kopp et al. 1998; Park et al. 2003). In oscillatory PCR systems, the sample is shunted back and forth between chambers that are held at different temperatures whereas closed-loop PCR chips utilize a thermosiphonic effect to move the sample around a fixed circuit. Both approaches offer flexibility in the number of thermal-cycles that are performed. In contrast, the number of cycles in a fixedloop system must be determined at the fabrication stage (see Fig. 1). Continuous-flow PCR 
devices generally require considerable optimization of the design and operating conditions to ensure the sample attains the appropriate temperatures and residence times for denaturation, annealing and extension.

To date, most continuous-flow PCR devices use a single-phase to fill the channel, which can lead to cross-contamination between successive samples, adsorption at the surface, and diffusional dilution of samples (e.g. Kopp et al. 1998). These problems can partially be overcome by making use of immiscible liquids to isolate the sample slugs from each other (Hardt et al. 2004). One factor that inhibits the PCR process in miniaturized devices can be related to surface interactions with the chip substrate. Droplet-based PCR offers the potential to eliminate this undesired contact between sample and substrate. Nisisako et al. (2002) have demonstrated that droplets of an aqueous phase can be dispersed into an immiscible oil phase. Each droplet potentially represents a transportable individual reaction volume that does not exchange material with its surroundings and the droplets will only touch the wall when required, and then only at specific locations. Droplet-based systems can therefore avoid the problems of adsorption, cross-contamination, and diffusional dilution associated with singlephase microfluidic systems.

A continuous-flow fixed-loop polymer chip has been constructed that takes advantage of droplet technology to perform real-time quantitative PCR. The samples are contained in aqueous droplets and continuously moved by an oil carrier-fluid through various temperature zones. In contrast to conventional continuous-flow devices which use a single aqueous phase with a larger thermal mass, this novel approach offers the prospect of lower thermal-cycling times and, as each sample is confined in a micro-droplet, any local temperature variations will be small and each droplet can achieve a more uniform temperature. The device can be employed with predetermined amounts of target nucleic acids, and can potentially be applied to single transcripts or used on single cells with the nucleic acids from one cell being amplified inside one droplet. Such an approach could greatly assist in transforming our understanding of how disease-associated transcripts relate to disease progression. As the samples are contained in droplets they could be detected and subsequently sorted on the chip itself, which is particularly important for an integrated lab-on-a-chip system.

This paper investigates many of the factors affecting droplet-based PCR chip design, including the thermal mass of the carrier-fluid and the substrate, the flow rate, and the thermal resistances of the various layers. The study employs both numerical and experimental approaches to analyze the fluid and substrate temperature distribution and the droplet 
residence times in critical zones. The paper highlights important thermal and transport design criteria for effective droplet-based PCR operation.

\section{PCR chip and optical detection system}

Figure 1 illustrates the continuous-flow fixed-loop PCR chip used in the present study. The overall dimensions of the device were $75 \times 75 \times 4 \mathrm{~mm}$ and the chips were fabricated from a polycarbonate sheet (RS, Corby, UK) using a precision milling machine (CAT3D-M6, Datron GmbH, Mühltal, Germany). The channels were sealed with a $100 \mu \mathrm{m}$ thick acetate foil which was attached to the polycarbonate chip using a thin film of UV-curable epoxy (Norland 68, Norland Products Inc., New Brunswick, NJ). To avoid blocking the channels, a hot roll laminator was used to spread the epoxy to a thickness of several microns between two sheets of acetate. The sheets were then placed on dry ice and cooled for 5 minutes before being separated. The solidified epoxy stays attached to one of the sheets allowing a seal with the chip. The chip and seal were then brought into contact with the use of the laminator and finally the epoxy was cured using a UV light source $\left(60-80 \mathrm{~mW} / \mathrm{cm}^{2}\right)$ for 2 minutes at a peak wavelength of $365 \mathrm{~nm}$. A $50 \mu \mathrm{m}$ diameter hole was drilled through the acetate sheet to allow the injection of the sample into the carrier-fluid. Various PCR chip designs were fabricated including devices with both planar and three-dimensional arrangements of heating channels. In the present study, we consider a planar two-temperature 32-cycle PCR chip as shown in Fig. 1. The serpentine loops are approximately $63 \mathrm{~mm}$ long (for one complete thermal cycle) and the distance between the centerlines of neighboring channels is $1 \mathrm{~mm}$. Details of the PCR chip layout and typical dimensions can be found in Figs. 2 and 3.

The introduction of the sample into the oil carrier-fluid is shown schematically in Fig. 4. The aqueous sample is extruded through a small orifice into the flowing oil where it breaks off into droplets that quickly become spherical due to surface tension. The droplets were produced in a $200 \mu \mathrm{m}$ wide by $200 \mu \mathrm{m}$ deep entrance channel which then tapered towards the main $500 \mu \mathrm{m}$ wide by $400 \mu \mathrm{m}$ deep heating channel. This design generates relatively high shear forces at the location where the aqueous samples are fed into the immiscible carrierfluid, thereby assisting the droplet formation process. At the same time, the design allows the flow velocity to be reduced in the main channel to prolong the droplet exposure to the different temperature zones. The resulting droplets are approximately spherical.

Experiments have been carried out to assess the relationship between the carrier-fluid flow rate and the droplet dimensions. The droplets are imaged, as shown in Fig. 5, so that their diameter and volume could be determined. For each flow rate, three video clips were recorded 
at 392-399 frames per second using a digital Firewire camera (PL-A741, monochrome 1.3 megapixel, PixeLink, Ottawa, Canada). The area of each droplet image was measured using an Object Analysis program developed in LabView (National Instruments, Austin, Texas, USA) and the average diameter and volume were calculated. For each clip, consisting of 500 frames, an average droplet diameter was obtained and the results are shown in Fig. 6. As clearly illustrated, the droplets can be reproduced with minimal size variation and the droplet size depends on the flow rates of the carrier-fluid and the sample. In the present experiments, using a constant sample flow rate of $4 \mu \mathrm{l} / \mathrm{min}$, the droplets vary between $100-155 \mu \mathrm{m}$ in diameter.

The standard PCR thermal profile consists of a denaturation step (368K), an annealing step (313-333K) and an extension step (345K). This is often referred to as a three-step thermal profile. To obtain high specificity, however, the annealing and extension temperatures can sometimes be merged, resulting in a two-stage thermal profile as described by Wittwer et al. (2001). In this case, the thermal profile consists of a denaturation step at $368 \mathrm{~K}$ and a combined annealing/extension step at between 333-345K. In the present PCR chip, two 50 Watt cartridge heaters (RS, Corby, UK) controlled by PID digital controllers (CAL 9900, RS, Corby, UK) are inserted into blocks of aluminum and positioned over the top surface of the chip as shown in Fig. 2. Since the positions of both heaters are flexible, the lengths of the channel in the different temperature zones can easily be adjusted to control the residence times in the denaturation and annealing/extension stages.

An array of GRIN (Gradient Refractive Index) lenses (Newport Spectra-Physics Ltd, Newbury, UK) are used to focus the excitation light into the flow channel, as shown in Fig. 7 which is for one optical element. The GRIN lenses have a radially-varying refractive index that causes the optical ray to follow a sinusoidal propagation path through the lens. This provides good light collection ability with a numerical aperture of 0.46 . In addition, the small dimensions of the lenses (diameter, $1.8 \mathrm{~mm}$; length $4.26 \mathrm{~mm}$ ) allow them to be inserted into one of the heater blocks avoiding a complicated optical arrangement. The correct focus of the lenses was determined by taking the different refractive indices of the air/seal and the seal/oil/sample into account. Reflection losses, which occur on any interface between materials of different refractive index, are kept to a minimum since the focused rays from the laser strike the droplet perpendicular to the oil/water interface as illustrated in Fig. 7. Emissions from the fluorophore-containing droplets are collected and collimated using the same GRIN lens. The emitted light is then separated from the excitation light through a $45^{\circ}$ dichroic mirror (Comar Instruments, Cambridge, UK) and finally imaged onto one of the 
elements of a multiple-channel photomultiplier (Hamamatsu, Japan). An achromatic imaging system composed of two convex lenses and one concave lens has been designed using WinLens 4.2 (Linos, Göttingen, Germany).

The laser beam can be split into 32 individual beams of equal intensity via an acrylic holographic beam splitter (Photonics and Analytical Marketing, Leeds, UK). The beams are collimated using a convex lens to match the GRIN lens array with a pitch of $2 \mathrm{~mm}$. With the exception of an $\mathrm{Ar}^{+}$- Laser (6 mW @ 488 nm, LaserGraphics, Germany), the chip and the optical system are housed in a light-tight box $(350 \times 230 \times 130 \mathrm{~mm})$ with all the crucial components mounted on an optical rail. Initial fluorescence measurements have been performed to test and calibrate the system. The optical system has the ability to detect the amount of fluorescence after every thermal cycle enabling quantitative real-time PCR measurements. An example of the fluorescence data from two neighboring detector elements is shown in Fig. 8.

The first step towards performing PCR in water-in-oil droplets is to develop a suitable PCR system that could initially be optimized on a conventional thermocycler. The RNase P gene is often selected as a common verification reference in multiplex DNA PCR reactions as it has the property of being a single-copy gene and therefore can be used to quantify the amplification product (Lee et al. 1991). The theoretical length of the template defined by the primers is 60 base pairs (bp). The primers were purchased from Sigma Genosys (Haverhill, UK) and the probes from Biosearch Technologies Inc. (Novato, CA, USA). The template DNA used in the PCR reactions is either human genome DNA or a specifically designed synthetic oligo replicating the RNase $\mathrm{P}$ sequence defined by the relevant two primers.

A Mastermix, GeneAmp ${ }^{\circledR}$ Fast PCR Master Mix (2x), was purchased from Applied Biosystems (Foster City, CA, USA). This Master Mix has "hot start" characterizations but does not need prolonged amplification. It has been optimized so that the PCR cycles can be performed with reduced time, which is particularly important because the droplets tend to merge at a flow rate less than $36 \mu \mathrm{L} / \mathrm{min}$. The samples were prepared using the $1 \mathrm{nM}$ oligo template and the GeneAmp® Fast PCR Master Mix. To heat activate the "hot start" DNA polymerase, the samples were preheated externally to $368 \mathrm{~K}$ for 10 minutes before being introduced into the chip to progress through the 32 thermal cycles. The residence time is between 5-8 s for both denaturation (368K) and annealing/extension (333-345K). The control and test samples were collected in an Eppendorf tube and analyzed by capillary gel electrophoresis using an Agilent 2100 Bioanalyzer. 
Figure 9 presents the results of a typical DNA amplification using the present PCR chip. The figure illustrates the fluorescence levels of the sample after 32 on-chip thermal cycles starting from a $1 \mathrm{nM}$ template with negligible initial fluorescence. The two markers correspond to 15 and 600 base pairs respectively, and the specifically amplified PCR fragment is a 60 base pair fragment from the RNase P gene. The results demonstrate the feasibility of the present chip design despite the fact that optimization of the system is still ongoing.

We have found that the main limiting factor of the present chip design is the residence time of the droplets in each temperature zone. At a production rate of approximately five droplets per second and a residence time exceeding 8 seconds, the droplets would merge before the end of the 32 cycles. Moreover, the performance of the PCR is significantly reduced once the annealing cycle time falls below approximately 5 seconds. As indicated in Fig. 10, the optimum residence time appears to lie between 5 and 8 seconds.

\section{Numerical model}

Thermal simulations of continuous-flow PCR devices have recently been reported by Zhang et al. (2002) and Sadler et al. (2003). The present investigation extends these studies by identifying the key factors affecting PCR chip design, including the specific heat capacity of the carrier-fluid, the flow rate, and the thermal resistances of the various layers making up the PCR chip.

The success of PCR can be related directly to whether the DNA samples achieve the specified denaturation and annealing/extension temperatures (Wittwer and Garling, 1991). However, direct measurement of the droplet temperature is extremely challenging at the present length scales and numerical simulation offers a convenient approach for assessing the temperature of the droplets.

The DNA samples are contained in aqueous droplets within an immiscible carrier-fluid (a light mineral oil or sunflower oil). Both oil and water are Newtonian fluids and therefore a linear stress/strain relationship can be assumed. In addition, the thermal conductivity and density of both liquids remain relatively constant over the PCR temperature range (333$368 \mathrm{~K})$. For example, the thermal conductivity and density of water are $0.66 \mathrm{~W} /(\mathrm{K} . \mathrm{m})$ and 983 $\mathrm{kg} / \mathrm{m}^{3}$ at $333 \mathrm{~K}$, while they are $0.68 \mathrm{~W} /(\mathrm{K} . \mathrm{m})$ and $962 \mathrm{~kg} / \mathrm{m}^{3}$ at $368 \mathrm{~K}$. The variations are less than $3 \%$ allowing average values of density and conductivity to be used in the numerical simulations. Similarly, the variations for the PCR oil carrier-fluid are less than $4 \%$. To further 
simplify the simulations, a constant viscosity has also been assumed. The physical properties of the materials used in the study can be found in Table 1 .

The governing equations can be written as follows:

Continuity equation:

$$
\frac{\partial \rho}{\partial t}+\nabla \cdot(\rho \boldsymbol{V})=0
$$

Momentum equation:

$$
\rho \frac{D \boldsymbol{V}}{D t}=-\nabla p+\mu \nabla^{2} \boldsymbol{V}+\rho \boldsymbol{g}
$$

Energy equation:

$$
\rho c_{\mathrm{p}} \frac{D T}{D t}=k \nabla^{2} T+\tau: \nabla \boldsymbol{V},
$$

where $D / D t$ is the substantive derivative and : represents the contraction operator of two tensors, $\rho$ is the fluid density, $\boldsymbol{V}$ is the velocity vector, $p$ is the pressure, $\mu$ is the dynamic viscosity, $\boldsymbol{g}$ is the acceleration due to gravity, $c_{\mathrm{p}}$ is the specific heat capacity, $T$ is the temperature and $k$ is the thermal conductivity. For a Newtonian fluid, the shear stress, $\tau$, is given by

$$
\tau=\mu \nabla \boldsymbol{V}
$$

For the heat transfer in the solid phase, the energy equation reduces to

$$
\rho c_{\mathrm{p}} \frac{\partial T}{\partial t}=k \nabla^{2} T .
$$

In the present study, the numerical simulations were conducted using the commercial Computational Fluid Dynamics code, CFD-ACE+ (ESI CFD, Huntsville, Alabama, USA). To reduce the computational cost, only six of the thermal cycles have been simulated.

The sample droplets in the PCR device are small in comparison to the heating channel and therefore they have minimal effect on the flow of the carrier-phase. Moreover, the droplets will respond quickly to any temperature changes in the carrier-fluid due to their large surfaceto-volume ratio. The droplet-laden liquid mixture can essentially be considered as a singlephase flow and only the carrier-fluid needs to be simulated.

\subsection{Boundary conditions}

Two constant temperature heaters are placed on the upper surface of the chip while the lower surface is exposed to air at room temperature, as shown schematically in Fig. 11. The temperatures of the two heaters were held constant at either $368 \mathrm{~K}$ for denaturation or $333 \mathrm{~K}$ for annealing/extension. The thermal resistance due to natural convection from the lower 
surface, $R_{3}$, has been estimated to be between $0.1 \sim 1.0 \mathrm{~m}^{2} . \mathrm{K} / \mathrm{W}$ (Zhang et al., 2002). The thermal resistance can be defined as $R=d / k$, where $d$ is the thickness of the layer and $k$ is the thermal conductivity. For the cellulose acetate (layer 1) and the polycarbonate (layer 2), $R_{1}$ and $R_{2}$, are several orders of magnitude smaller than $R_{3}$. This implies that the most significant drop in temperature occurs at the polycarbonate-air interface at the base of the chip. Layer 3 will have only a minimal effect on the temperature distribution in the channel and therefore an adiabatic boundary condition can be assumed on the lower surface. At the inlet, the fluid is preheated to $368 \mathrm{~K}$ and a constant flow rate is imposed. In addition, a no-slip boundary condition has been assumed along the walls of the channel.

\section{Results and discussion}

The PCR chip design and operating conditions need to be optimized to ensure the droplets achieve and maintain the desired temperatures for between 5 and 8 seconds during each thermal cycle. In addition, the time required for the heating and cooling stages should be minimized to improve the throughput of the device.

In each thermal cycle, the carrier-fluid moves through a high and a low temperature region, maintained at $368 \mathrm{~K}$ and $333 \mathrm{~K}$, respectively, followed by a cooling and heating transition region. Figure 12(a) shows that the predicted heating and cooling zones are relatively short and the desired temperatures can be readily achieved when the fluid is stationary. However, once the fluid starts to move, thermal energy will be carried by the moving fluid and the heating and cooling zones become more noticeable, as illustrated in Fig. 12(b).

Figure 13 shows the predicted temperature distribution along the centerline of the channel over one representative thermal cycle. The thermal inertia of the moving fluid causes an effective reduction in the length of the denaturation and annealing/extension zones. This will have a significant impact on the design and optimization of PCR operating conditions and will clearly affect the yield. For example, if the same flow rate needs to be maintained, the serpentine loop should be longer to achieve the desired residence time.

The effect of fluid transport on the temperature field has been visualized qualitatively using a thermally sensitive dye, Kromagen thermochromic ink (Thermographic Measurement Ltd, Flintshire, UK). On the heating cycle, the dye starts to lose color at $339.2 \mathrm{~K}$ and becomes colorless at $341.3 \mathrm{~K}$. On the cooling cycle, the color starts to return at $339.8 \mathrm{~K}$ and fully recovers at $336.6 \mathrm{~K}$. Figure 14 clearly shows that the dye transition point lies between the two 
heating blocks when the fluid is stationary but will move downstream as the flow rate increases.

The thermal mass, characterized by the product $\rho c_{\mathrm{p}}$, is a measure of the ability of a material to store thermal energy. A moving fluid with a high thermal mass will transport more thermal energy than a fluid with low thermal mass and it will have a larger impact on the temperature of the surrounding medium. The choice of oil or water as the working fluid will lead to different temperature distributions in the channel. Water has a larger thermal mass than oil and consequently the thermal development length (the distance a fluid travels to achieve the same temperature as the contacting surface) will be longer. Figure 15 shows the predicted centerline temperature distributions over the sixth complete cycle. At a flow rate of 120 $\mu 1 /$ min, chosen to amplify inertial effects, the thermal development length of water prevents it from reaching the desired operating temperatures of $368 \mathrm{~K}$ and $333 \mathrm{~K}$. In contrast, it is clear that oil responds to the surrounding temperature field more rapidly than water because of its lower thermal mass and is therefore able to be closer to the desired operating temperatures. The coupled nature of the solid-fluid thermal interaction means that the thermal development length is not simply a product of the Prandtl and Reynolds numbers, as found in the classic Graetz problem. Ideally, the carrier-fluid should have a low thermal mass to ensure that the heating and cooling times are minimized.

A simple test case will be used to illustrate how the thermal resistance of the acetate layer affects the temperature distribution in the working fluid. As previously discussed, the adiabatic boundary condition on the lower surface of the chip can be justified when $R_{1}<<R_{3}$. $\mathrm{R}_{1}, \mathrm{R}_{2}$ and $\mathrm{R}_{3}$ are the thermal resistances of the cellulose acetate layer, the polycarbonate layer and air, respectively. Maintaining the adiabatic assumption for $R_{3}$, the impact of $R_{1}$ can be assessed by varying either the material's thermal conductivity or the thickness of the layer. Changes to the value of $R_{1}$ can have a significant impact on the temperature field, as shown in Fig. 16 , where $R_{1}$ increases from $5 \times 10^{-4}$ to $5 \times 10^{-3} \mathrm{~m}^{2} . \mathrm{K} / \mathrm{W}$ while $R_{2}$ remains constant at $1.38 \times 10^{-2} \mathrm{~m}^{2} . \mathrm{K} / \mathrm{W}$. In this example, with oil as the working fluid, the performance is clearly improved when the thermal resistance of layer 1 is small. Therefore, in addition to the low thermal mass requirement, it is important to ensure that $R_{1}$ is much less than either $R_{2}$ or $R_{3}$.

The flow rate clearly has a direct impact on the droplet residence time and strongly affects the carrier-fluid's temperature distribution. Figure 17 examines the effect of changing the flow rate of the oil carrier-fluid. When the flow rate is low, the heating and cooling zones are short and the PCR operating temperatures are achieved and maintained for a significant proportion 
of the thermal cycle. Increasing the flow rate raises the thermal inertia of the fluid and this has a significant influence on the temperature field in the channel. When the flow rate exceeds 60 $\mu \mathrm{l} / \mathrm{min}$, the proportion of time spent in the heating and cooling regions starts to become significant. Moreover, above a flow rate of $120 \mu \mathrm{l} / \mathrm{min}$, the oil fails to reach the required denaturation and annealing/extension temperatures. For this particular chip design, flow rates above $120 \mu \mathrm{l} / \mathrm{min}$ need to be avoided for successful PCR operation.

The previous analysis has focused on the temperature distribution of the carrier-fluid. However, for droplet-based PCR, we need to ensure that the reagents contained in the water droplet spend sufficient time at the denaturation and annealing/extension temperatures. At a given flow rate, the droplet's residence time will depend on the final velocity achieved by the droplet. This velocity will depend on the confining influence of the channel walls and the vertical position of the droplet due to buoyancy and lift forces. Neutrally buoyant droplets will travel along the centerline of the channel but will not travel at the peak velocity. The confining influence of the channel strongly affects the drag experienced by the droplet which will act to reduce its final velocity. In general, the vertical location of the droplet will be determined by a balance between buoyancy and hydrodynamic lift forces. A water droplet immersed in an oil carrier-fluid will experience a force due to buoyancy, $f_{b}$, given by

$$
f_{b}=\frac{\pi}{6} d^{3} \Delta \rho g,
$$

where $\Delta \rho$ is the density difference between the droplet and the carrier-fluid. The hydrodynamic lift force is related to the velocity gradient and can be estimated (Saffman, 1965) from

$$
f_{\text {Saff }}=1.61 \mu d\left|V_{r}\right| \sqrt{\operatorname{Re}}
$$

where the droplet Reynolds number is $R e=\rho d^{2}|\nabla V| / \mu, V_{r}$ is the relative velocity between the droplet and the carrier-fluid, and $\nabla V$ is the velocity gradient in the carrier-fluid. One factor that needs to be considered in determining residence time is the density difference due to temperature variation and any resulting buoyancy force. To prevent the droplets making contact with the surface it is better to choose fluids with similar densities. In practice, large shear rates in the near-wall region help to prevent the droplets making physical contact with the surface, especially at high flow rates.

In the present PCR chip design, the channel is $500 \mu \mathrm{m}$ wide and $400 \mu \mathrm{m}$ deep, and the droplets are typically $100 \mu \mathrm{m}$ in diameter. A flow rate of $120 \mu \mathrm{l} / \mathrm{min}$ corresponds to an average velocity of $0.01 \mathrm{~m} / \mathrm{s}$. Under these conditions, the buoyancy force is larger than the Saffman lift force even near the walls of the channel. The water droplet will therefore be 
transported close to the wall and, as a consequence, will move more slowly than the oil in the center of the channel. An estimate of the droplet speed can be obtained by integrating the velocity over the cross-sectional area occupied by the droplet. For a flow rate of $60 \mu 1 / \mathrm{min}$, the residence time of the droplet in the denaturation zone is about 4.8 seconds while the time spent in the annealing/extension zone is approximately 4.4 seconds. As the desired residence time for the droplet is between 5 and 8 seconds, it is likely that a flow rate of $60 \mu \mathrm{l} / \mathrm{min}$ will be too high for efficient DNA amplification. The PCR yield can be described theoretically by $(1+\gamma)^{n}$, where $\gamma$ is the amplification efficiency and $n$ the number of cycles. Unfavorable operating conditions will lead to decreased DNA strand separation and a lower than optimum temperature for DNA polymerase activity which will result in a low value of $\gamma$ and, consequently, a poor PCR yield.

\section{Conclusions}

This paper has investigated many of the factors affecting droplet-based PCR chip design, including the thermal mass of the carrier-fluid, the flow rate, and the thermal resistance of the different materials. The success of droplet-based PCR is crucially dependent on whether the samples achieve the specified temperatures for denaturation and annealing/extension. Due to the difficulties associated with measuring droplet temperatures, an extensive numerical study has been performed to understand the important design criteria. The study highlights many issues that need to be considered for successful PCR operation. Ideally, the carrier-fluid should have a low thermal mass to ensure that the heating and cooling time is minimized. Numerical simulations have indicated that the solid-fluid thermal interaction within the chip is subtle and the thermal development length is not simply a product of the Prandtl and Reynolds numbers, as observed in the standard Graetz problem.

As expected, the flow rate has a major influence on the droplet residence time and strongly affects the carrier-fluid's temperature distribution. When the flow rate exceeds a critical value, the carrier-fluid will fail to reach the stipulated design temperatures. It has also been shown that the thermal resistance of the various layers could adversely affect the temperature distribution in the channel.

Droplet-based PCR offers many advantages over traditional well-based amplification techniques with the possibility of ultra-high throughput being combined with sophisticated separation, control, and analysis on a single chip. Moreover, droplet-based systems can avoid many of the problems associated with single-phase PCR techniques, including adsorption, cross-contamination, and diffusional dilution of the sample. The very high throughput rate of 
droplet-based PCR also has the advantage of uniting the sample preparation and the PCR stages. This is particularly important for controlling the amount of template entering a droplet by dilution, which enhances subsequent quantitative analyses. However, it has been shown that many factors need to be considered in this novel type of PCR design. 


\section{Acknowledgments}

The authors are grateful to the UK Engineering and Physical Sciences Research Council (EPSRC) for supporting this research under grant No. GR/S82978/01. Additional support was provided by EPSRC under the auspices of Collaborative Computational Project 12 (CCP12). Thermographic Measurement Ltd, Flintshire, UK is also acknowledged for supplying samples of the thermochromic dye.

\section{References}

Auroux PA, Iossifidis D, Reyes DR, Manz A (2002) Micro total analysis systems 2: Analytical standard operations and applications. Anal. Chem. 74:2637-2652

Auroux PA, Koc Y, deMello A, Manz A, Day PJR (2004) Miniaturised nucleic acid analysis. Lab Chip 4:534-546

Auroux PA, Day PJR, Manz A (2005) Quantitative study of the adsorption of PCR reagents during on-chip bi-directional shunting PCR. $9^{\text {th }}$ Intl. Conf. on Miniaturized Systems for Chemistry and Life Sciences, Boston, Massachusetts, USA

Belgrader P, Benett W, Hadley D, Richards J, Stratton P, Mariella R, Milanovich F (1999) Infectious disease: PCR detection of bacteria in seven minutes. Science 284:449-450

Bu MQ, Tracy M, Ensell G, Wilkinson JS, Evans AGR (2003) Design and theoretical evaluation of a novel microfluidic device to be used for PCR. J. Micromech. Microeng. 13: S125-S130

CFD-ACE+ User Manual Version 2006, ESI CFD Inc., Huntsville, AL 35806, USA

Chen ZY, Qian SZ, Abrams WR, Malamud D, Bau HH (2004) Thermosiphon-based PCR reactor: experiment and modeling. Anal. Chem. 76:3707-3715

Chiou J, Matsudaira P, Sonin A, Ehrlich D (2001), A closed-cycle capillary polymerase chain reaction machine. Anal. Chem. 73:2018-2021

Gulliksen A, Solli L, Karlsen F, Rogne H, Hovig E, Nordstrom T, Sirevag R (2004) Real-time nucleic acid sequence-based amplification in nanoliter volumes. Anal. Chem. 76:9-14

Hardt S, Dadic D, Doffing F, Drese KS, Münchow G, Sörensen O (2004) Development of a slug-flow PCR chip with minimum heating cycle times. Nanotech. 1:55-58 
Kopp MU, deMello AJ, Manz A (1998) Chemical amplification: continuous-flow PCR on a chip. Science 280:1046-1048

Lee JY, Rohlman CE, Molony LA, Engelke DR (1991) Characterization of RPR1, an essential gene encoding the RNA component of saccharomyces-cerevisiae nuclear RNASE-P. Mol. Cell. Biol. 11(2):721-730

Liu J, Enzelberger M, Quake S (2002) A nanoliter rotary device for polymerase chain reaction. Electrophoresis 23:1531-1536

Nakano H, Matsuda K, Yohda M, Nagamune T, Endo I, Yamane T (1994) High-speed polymerase chain-reaction in constant flow. Biosci. Biotechnol. Biochem. 58:349-352

Nisisako T, Torii T, Higuchi T (2002) Droplet formation in a microchannel network. Lab Chip 2:24-26

Northrup MA, Ching MT, White RM, Watson RT (1993) DNA amplification with a microfabricated reaction chamber. Transducers'93:924-926

Park N, Kim S, Hahn JH (2003) Cylindrical compact thermal-cycling device for continuousflow polymerase chain reaction. Anal. Chem. 75:6029-6033

Sadler DJ, Changrani R, Roberts P, Chou CF, Zenhausern F (2003) Thermal management of bioMEMS: temperature control for ceramic-based PCR and DNA detection devices. IEEE Trans. Compon. Packag. Technol. 26:309-316

Saffman PG (1965) The lift on a small sphere in a slow shear flow. J. Fluid Mech. 22:385400. Corrigendum (1968) 31:624

Saiki RK, Scharf S, Faloona F, Mullis KB, Horn GT, Erlich HA, Arnheim N (1985) Enzymatic amplification of betaglobin genomic sequences and restriction site analysis for diagnosis of sickle-cell anemia. Science 230:1350-1354

Vilkner T, Janasek D, Manz A (2004) Micro total analysis systems: Recent developments. Anal. Chem. 76:3373-3385

West J, Karamata B, Lillis B, Gleeson JP, Alderman J, Collins JK, Lane W, Mathewson A, Berney H (2002) Application of magnetohydrodynamic actuation to continuous flow chemistry. Lab Chip 2: 224-230 
Wilding P, Shoffner MA, Kricka LJ (1994) PCR in silicon microstructure. Clin. Chem. 40:1815-1818

Wittwer CT, Garling DJ (1991) Rapid cycle DNA amplification: time and temperature optimisation. BioTechniques 10:76-83

Wittwer CT, Herrmann MG, Gundry CN, Elenitoba-Johnson KSJ (2001) Real-time multiplex PCR assays. Methods 25: 430-442

Zhang Q, Wang W, Zhang H, Wang Y (2002) Temperature analysis of continuous-flow micro-PCR based on FEA. Sens. Actuators B 82:75-81 


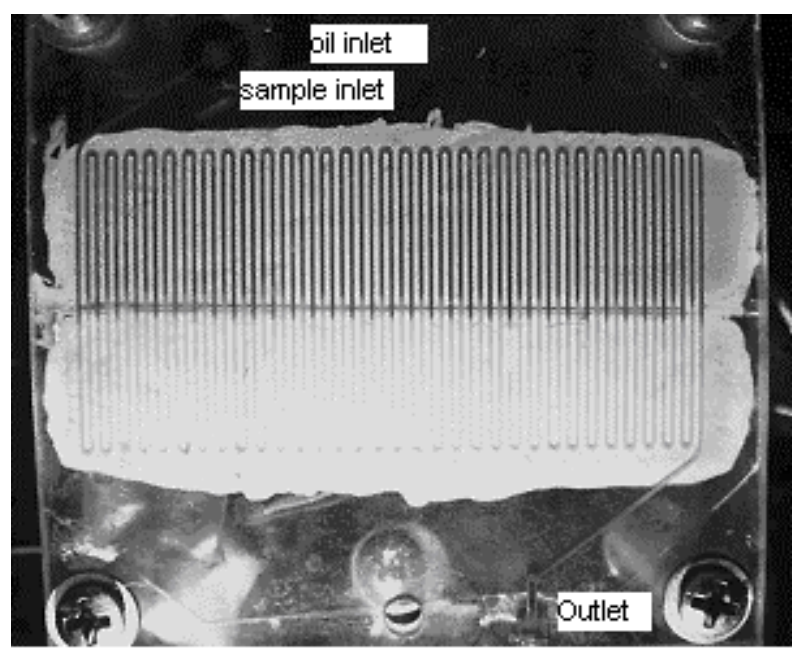

Fig. 1 The 32-cycle continuous-flow PCR chip used in the present study. The image shows an experimental visualization of the temperature distribution within the chip using Kromagen thermochromic dye with an approximate transition temperature of $340.5 \mathrm{~K}$ (Thermographic Measurement Ltd, Flintshire, UK). The dye becomes brighter (colorless) when the temperature increases and darker when the temperature decreases. 


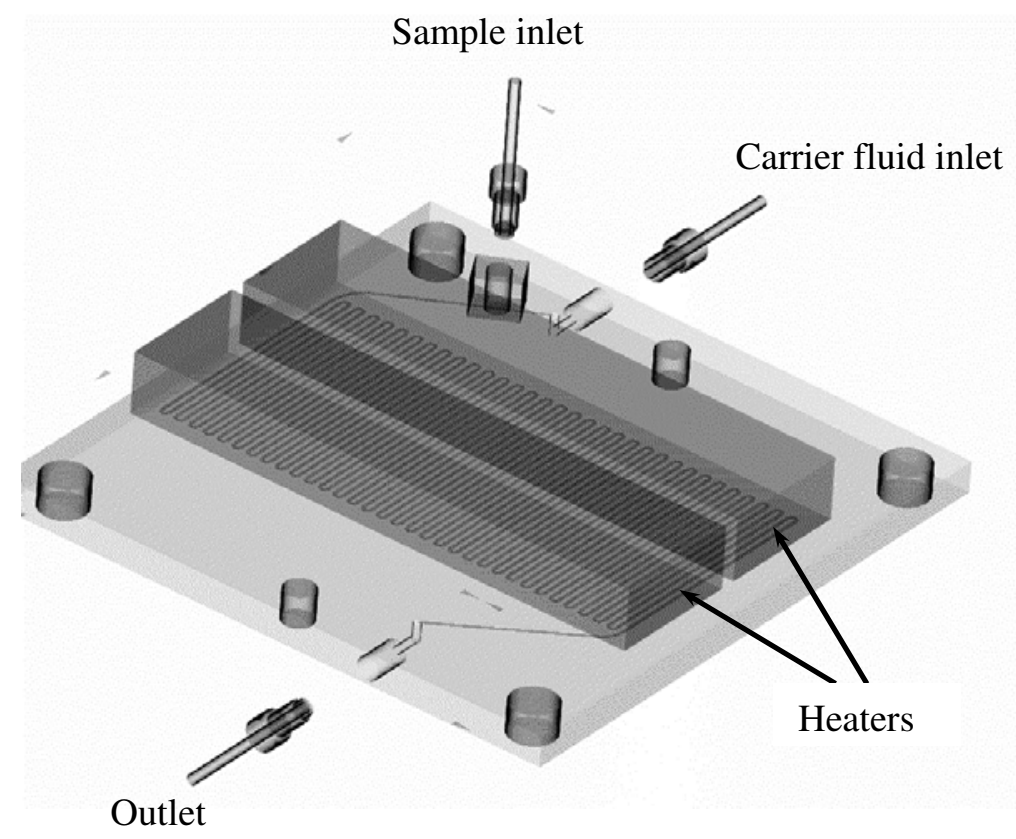

Fig. 2 Schematic representation of the PCR chip showing the overall layout and the inlets and outlet. 


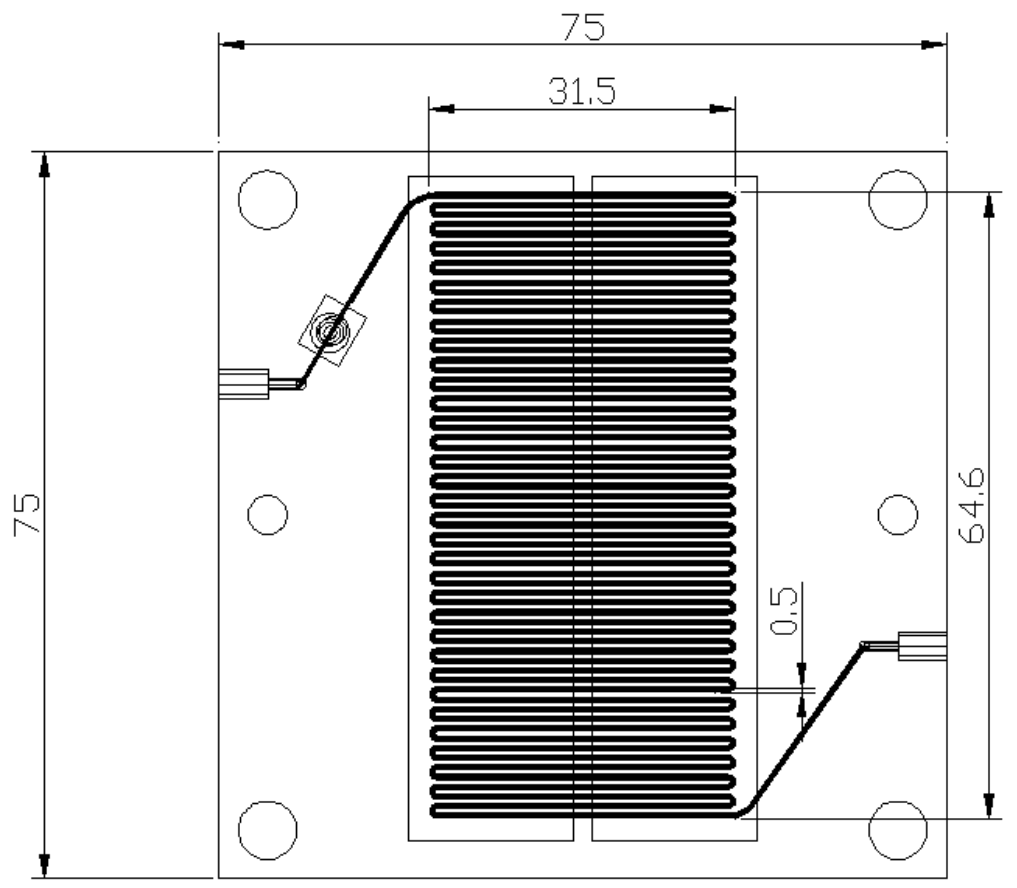

Fig. 3 Dimensions of the PCR chip (in mm). The channel depth is $400 \mu \mathrm{m}$.

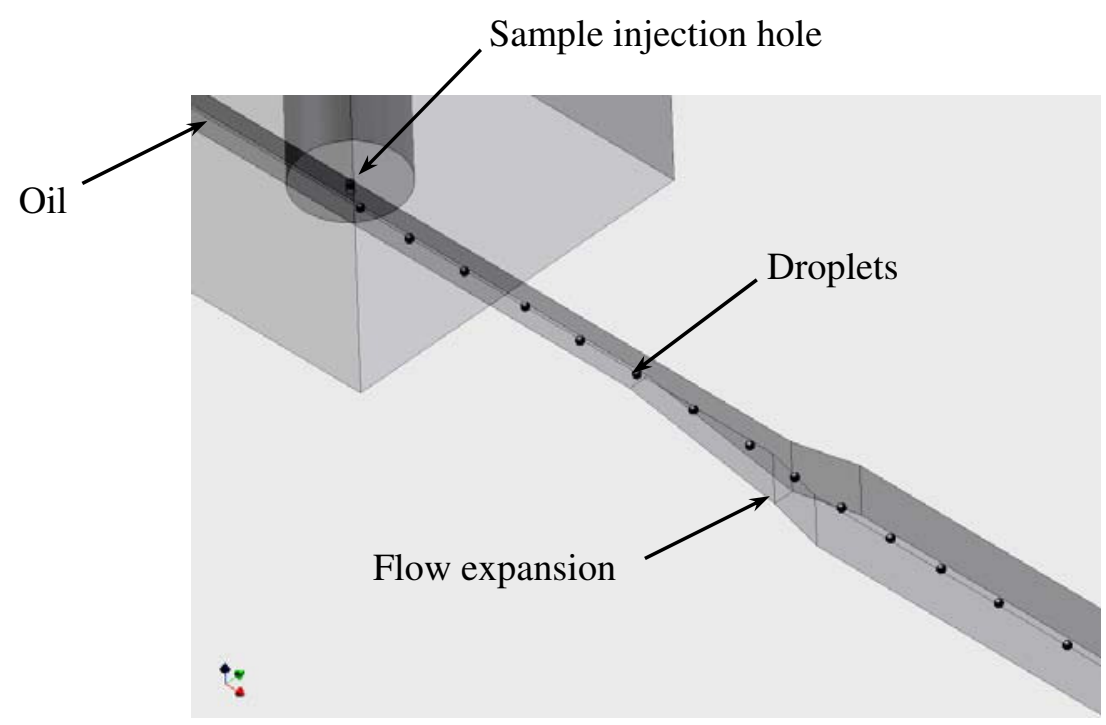

Fig. 4 Detail of the tapered region of the PCR chip where the sample is introduced into the oil phase. The sample is extruded through a $50 \mu \mathrm{m}$ diameter orifice into the flowing oil where it breaks off into droplets that quickly become spherical due to surface tension. 


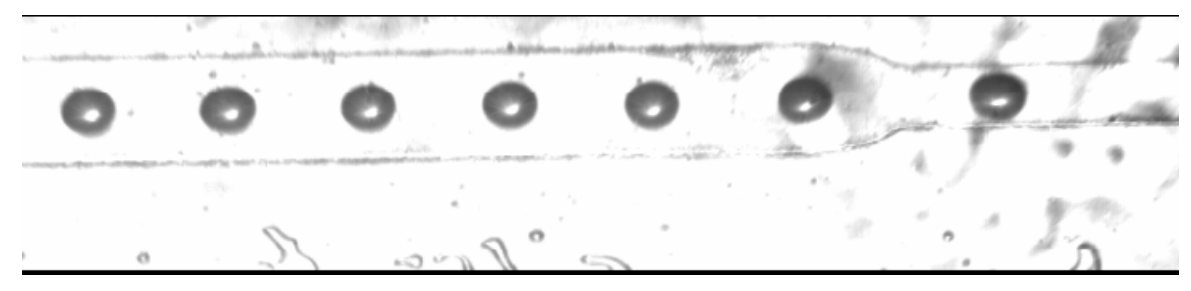

Fig. 5 A typical frame from the video clip being analyzed.

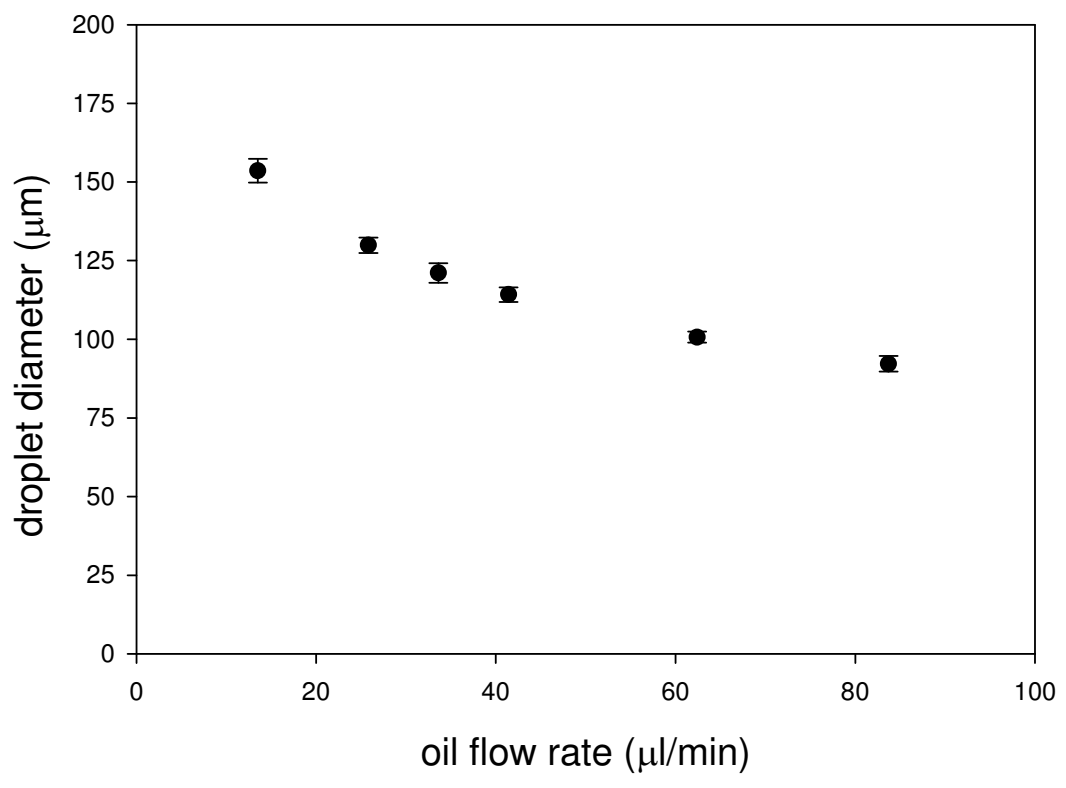

Fig. 6 The effect of oil flow rate on the average droplet size for a constant sample flow rate of $4 \mu \mathrm{l} / \mathrm{min}$. The error bars represent the standard deviation in droplet size. 


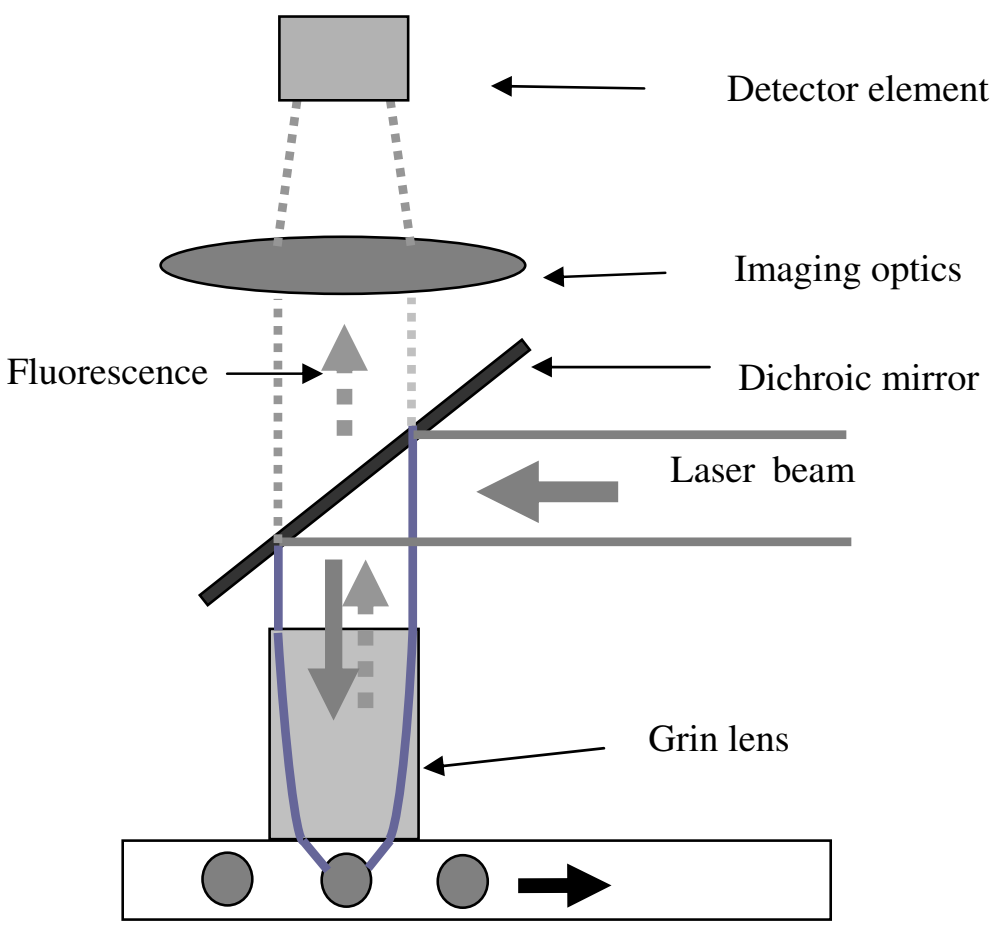

Fig. 7 Schematic diagram of the optical detection system showing a single element of the GRIN lens array. The GRIN lenses are inserted in the low temperature heater element and are in direct contact with the surface of the flow chip.

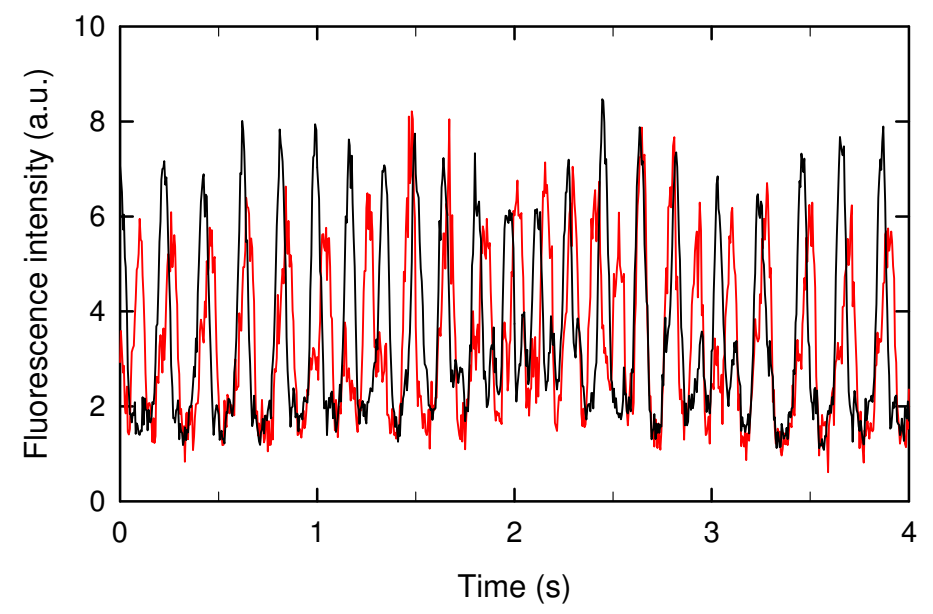

Fig. 8 Fluorescence data from two neighboring detector elements. Each peak represents a single droplet. 


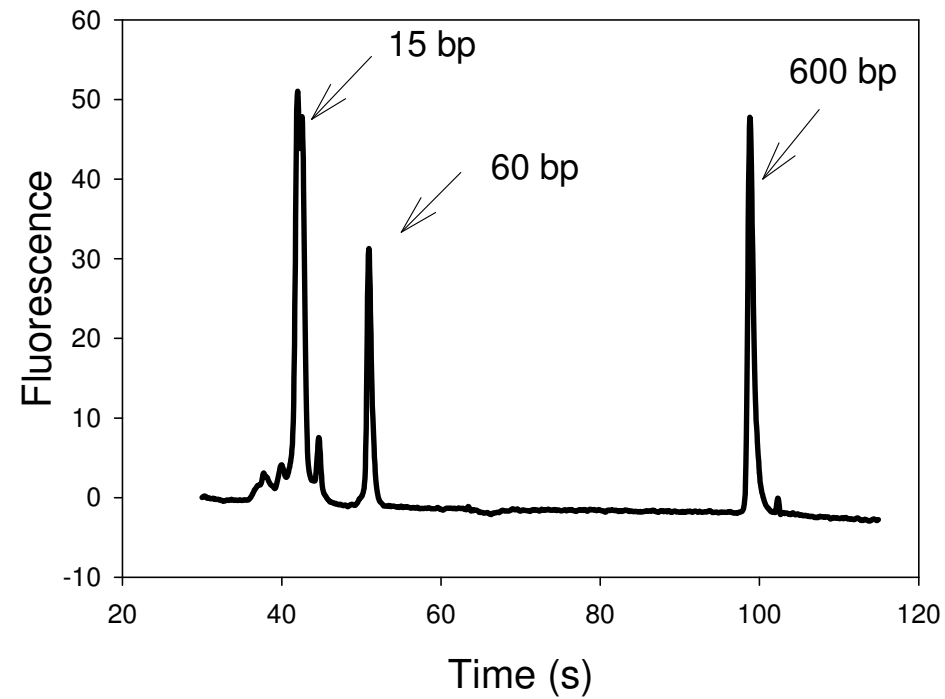

Fig. 9 Samples from the chip were analyzed by gel electrophoresis using an Agilent 2100 Bioanalyzer. The two peaks with 15 and 600 base pairs (bp) are markers added prior to analysis. The specifically amplified PCR fragment is a 60 base pair fragment from the RNase P gene.

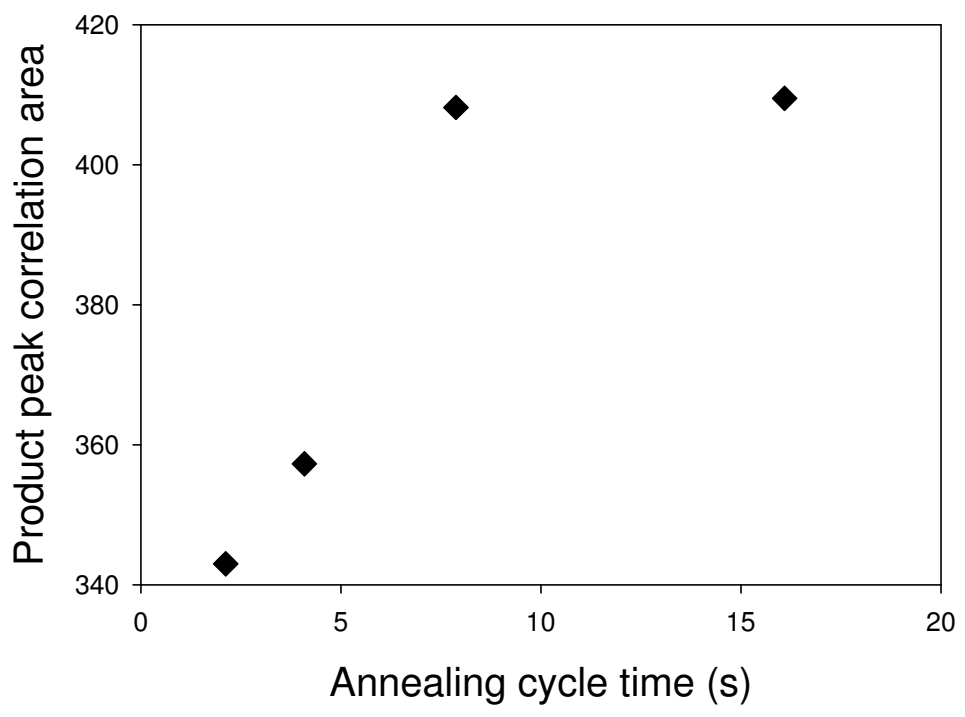

Fig. 10 The efficiency of GeneAmp® Fast PCR Master Mix with varying residence time for annealing/extension as measured using a conventional thermocycler. The PCR performance drops exponentially once the residence time falls below $7 \mathrm{~s}$. 


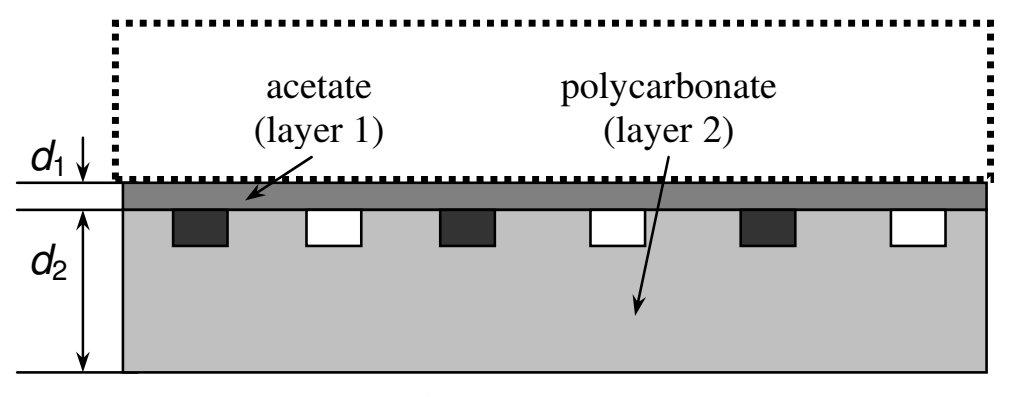

air (layer 3)

Fig. 11 Schematic diagram of the cross-section through 3 representative cycles of the PCR chip. The black and white rectangles denote the fluidic channels. Two heater elements, shown as a dashed box, are positioned on the upper surface of the chip and are maintained at a uniform temperature of $368 \mathrm{~K}$ for the denaturation stage and $333 \mathrm{~K}$ for the annealing/extension stage. 


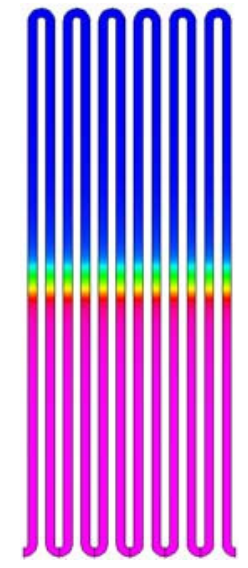

(a)
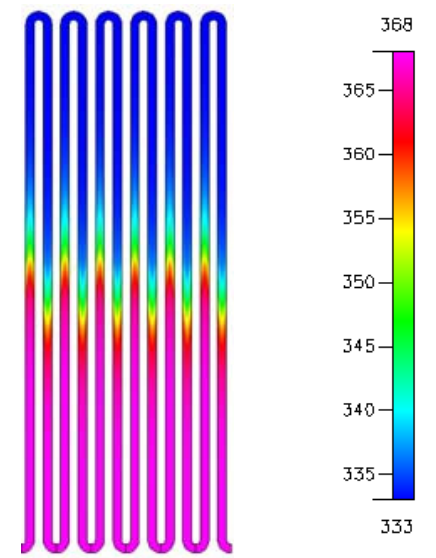

$T(\mathrm{~K})$

(b)

Fig. 12 Predicted temperature distribution of the oil carrier-fluid over six thermal cycles for two different flow rates, (a) $Q=0$, and (b) $Q=60 \mu \mathrm{l} / \mathrm{min}$.

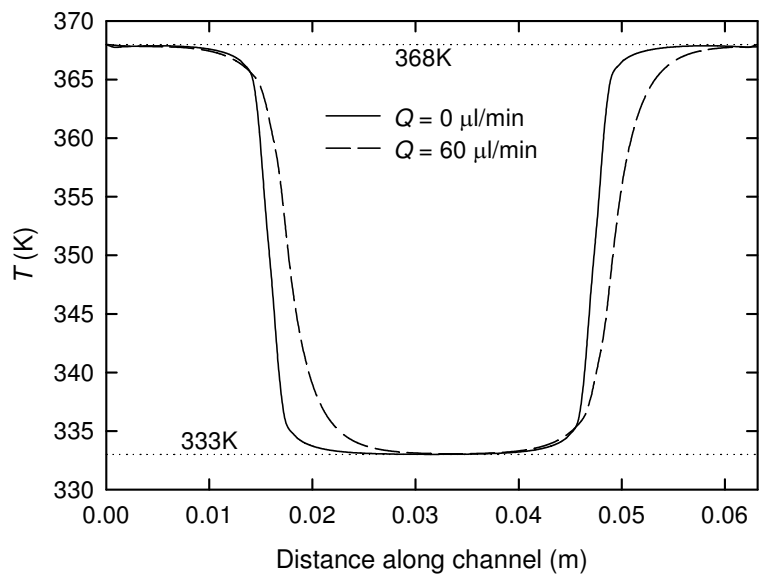

Fig. 13 Temperature distribution of the oil carrier-fluid along the centerline of the channel. The thermal resistances of the acetate and polycarbonate layers are taken to be $R_{1}=5 \times 10^{-4}$ $\mathrm{m}^{2} . \mathrm{K} / \mathrm{W}$, and $R_{2}=1.38 \times 10^{-2} \mathrm{~m}^{2} . \mathrm{K} / \mathrm{W}$. 


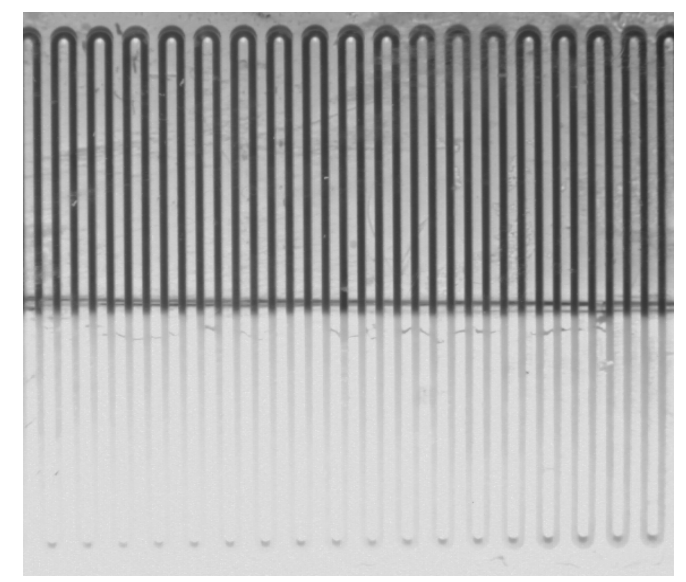

(a)

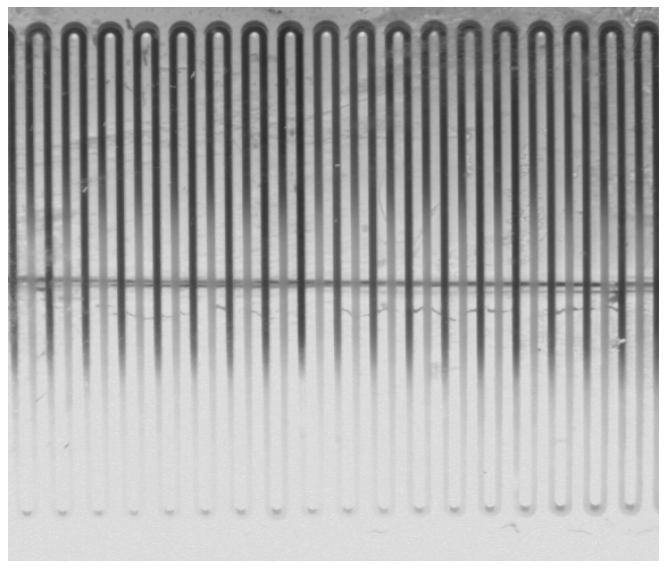

(b)

Fig. 14 Experimental visualization of the temperature distribution along the channel at two different flow rates, (a) $Q=0$, and (b) $Q=51 \mu \mathrm{l} / \mathrm{min}$. The distribution of the temperature was imaged using Kromagen thermal ink with an approximate transition temperature of $340.5 \mathrm{~K}$. The dye becomes colorless when the temperature increases and darker when the temperature decreases. 


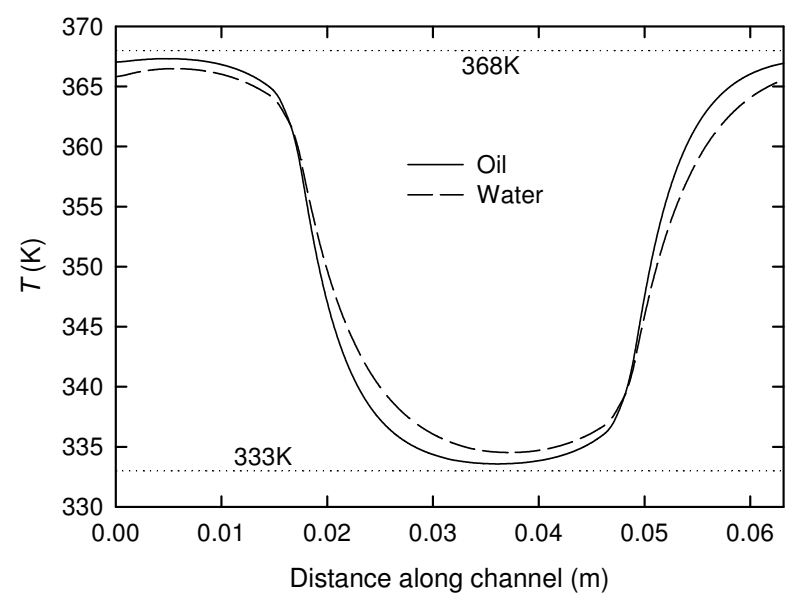

Fig. 15 Effect of using different working fluids on the temperature profile along the centerline of the channel for a flow rate of $Q=120 \mu \mathrm{l} / \mathrm{min}$. The thermal resistances of the acetate and polycarbonate layers are taken to be $R_{1}=5 \times 10^{-4} \mathrm{~m}^{2} . \mathrm{K} / \mathrm{W}$, and $R_{2}=1.38 \times 10^{-2} \mathrm{~m}^{2} . \mathrm{K} / \mathrm{W}$.

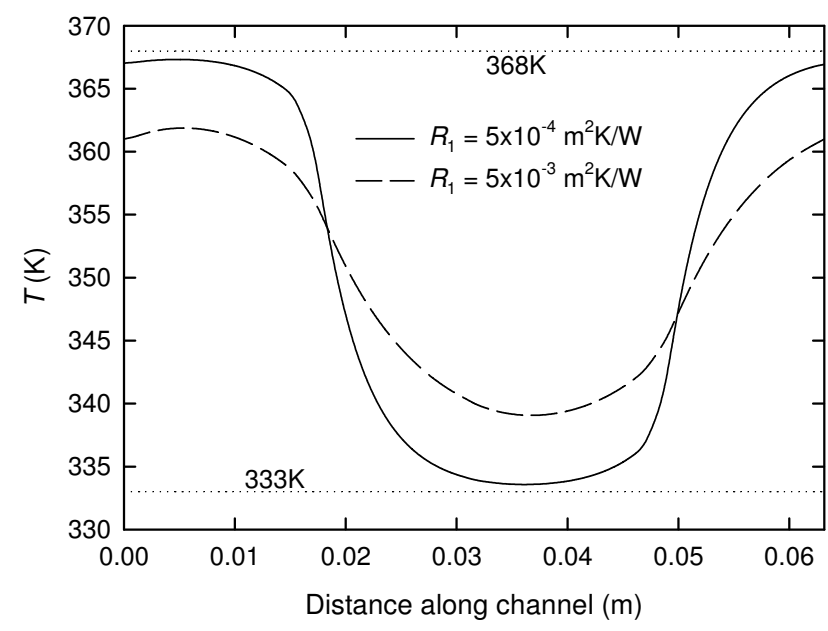

Fig. 16 Effect of varying the thermal resistance of layer 1 on the temperature profile along the centerline of the channel. The working fluid is oil, $Q=120 \mu \mathrm{l} / \mathrm{min}$, and the thermal resistance of the polycarbonate layer, $R_{2}=1.38 \times 10^{-2} \mathrm{~m}^{2} . \mathrm{K} / \mathrm{W}$. 


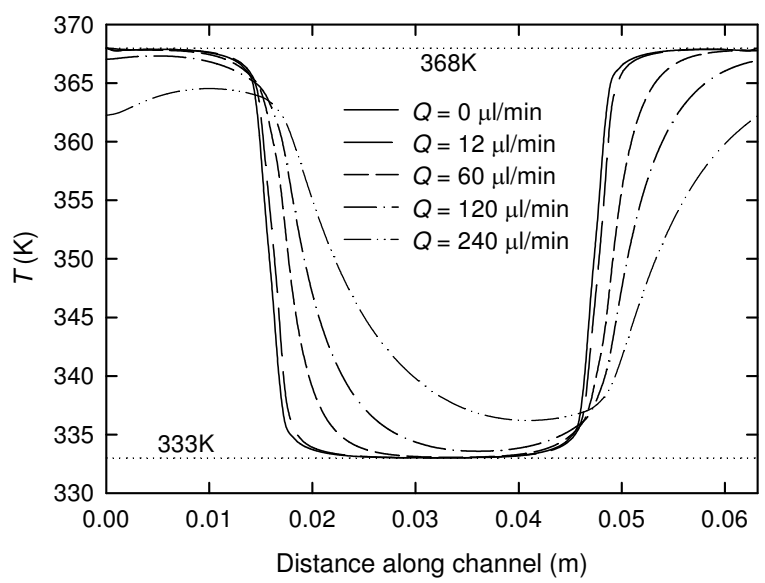

Fig. 17 Influence of the flow rate on the temperature profile along the centerline of the channel. The carrier-fluid is oil, $R_{1}=5 \times 10^{-4} \mathrm{~m}^{2} . \mathrm{K} / \mathrm{W}$ and $R_{2}=1.38 \times 10^{-2} \mathrm{~m}^{2} . \mathrm{K} / \mathrm{W}$. 
Table 1 Material properties of the PCR chip.

\begin{tabular}{|c|c|c|c|c|}
\hline & $\rho$ & $k$ & $c_{p} \times 10^{-3}$ & $v \times 10^{6}$ \\
\hline Polycarbonate & 1200 & 0.2884 & 1.256 & - \\
\hline Cellulose acetate & $1260-1300$ & 0.20 & 1.4 & - \\
\hline Oil & $915-918$ & 0.17 & 2.0 & 9.4 \\
\hline Water & 979 & 0.67 & 4.2 & 0.4 \\
\hline
\end{tabular}

